\title{
Philonsorbonne
}

$10 \mid 2016$

Année 2015-2016

\section{Le débat sur le statut des corps dans la philosophie de Descartes}

\section{Mariana DE ALMEIDA CAMPOS}

\section{OpenEdition}

1 Journals

Édition électronique

URL : https://journals.openedition.org/philonsorbonne/774

DOI : 10.4000/philonsorbonne.774

ISSN : 2270-7336

Éditeur

Publications de la Sorbonne

Édition imprimée

Date de publication : 1 janvier 2016

Pagination : $29-47$

ISSN : 1255-183X

\section{Référence électronique}

Mariana DE ALMEIDA CAMPOS, «Le débat sur le statut des corps dans la philosophie de Descartes », Philonsorbonne [En ligne], 10 | 2016, mis en ligne le 19 janvier 2016, consulté le 08 juin 2021. URL : http://journals.openedition.org/philonsorbonne/774; DOI : https://doi.org/10.4000/philonsorbonne. 774

(c) Tous droits réservés 


\title{
Le débat sur le statut des corps dans la philosophie de Descartes
}

\author{
Mariana DE ALMEIDA CAMPOS
}

\section{Introduction}

Lorsque nous nous demandons si Descartes admet une seule substance corporelle ou une pluralité de substances corporelles, la réponse à cette question n'est pas simple. Dans la Méditation Troisième, il dit que la pierre est une substance : "Car, lorsque je pense qu'une pierre est une substance, ou bien une chose qui de soi est capable d'exister, puis que je suis une substance, quoique je conçoive bien que je suis une chose qui pense et non étendue, et que la pierre au contraire est une chose étendue et qui ne pense point, et qu'ainsi entre ces deux conceptions il se rencontre une notable différence, toutefois elles semblent convenir en ce qu'elles représentent des substances $»^{1}$. Cependant, dans un fragment conservé par Baillet d'une lettre écrite pendant l'été 1631 à Villebressieu, chimiste et philosophe naturel, Descartes affirme qu'il existe une seule substance corporelle dans la nature : «Il me semble même que vous avez déjà découvert des généralités de la nature, comme : qu'il n'y a qu'une seule substance matérielle, qui reçoit d'un agent externe l'action ou le moyen de se mouvoir localement, d'où elle tire diverses figures ou modes, qui la rendent telle que nous la voyons dans ces premiers composés que l'on appelle les Éléments [...] Ce qui cadre beaucoup avec ma manière de philosopher, et qui revient merveilleusement à toutes les expériences mécaniques que j'ai faites de la nature sur ce sujet $»^{2}$. Ainsi, en se basant sur les textes, les deux positions pourraient apparemment être considérées comme exprimant une position cartésienne.

1. L'édition des textes de Descartes à laquelle nous ferons référence est celle des Euvres de Descartes en 12 volumes, C. Adam et P. Tannery (éds), Paris, Librairie Philosophique J. Vrin, 1996. AT, VII, 44. 
Dans le présent article, nous essayerons de déterminer quelle serait, précisément, la position de Descartes sur le nombre des substances corporelles, et par conséquent sur le statut ontologique des corps. Les substances corporelles sont-elles plusieurs ou une seule ? Les corps sont-ils des substances ou des modes? Lorsque nous examinerons ces questions, nous montrerons qu'elles s'insèrent dans un débat existant dans la littérature sur Descartes qui sépare les interprètes entre pluralistes et monistes. En considérant ce débat, nous présenterons d'abord les bases textuelles de l'interprétation pluraliste, ainsi que ses principaux arguments. Ensuite, nous évoquerons quelques difficultés rencontrées dans cette interprétation. Nous terminerons en essayant d'évaluer le résultat obtenu et en proposant l'interprétation moniste comme issue. Au moment d'examiner cette dernière interprétation, nous attirerons néanmoins l'attention sur un problème qui se pose à elle, mais que nous croyons pouvoir être résolu.

\section{L'interprétation pluraliste}

Nous appelons pluraliste la vision selon laquelle il existe une pluralité de substances corporelles dans la nature ${ }^{3}$. Il existe de nombreux textes dans lesquels Descartes dénomme les corps particuliers substances. Comme nous avons indiqué précédemment, dans la Méditation Troisième, par exemple, il dit que la pierre est une substance ${ }^{4}$. Ensuite, dans l'article 61 de la $1^{\text {re }}$ partie des Principes, il se réfère également à la pierre comme si elle était une substance où des modes, tels que la forme et le mouvement, sont inhérents ${ }^{5}$. En plus, dans les Réponses aux Quatrièmes Objections, il affirme que la main et le bras d'un homme sont des substances ${ }^{6}$. Puis, dans les Sixièmes Réponses, il considère les os et la chair d'un animal comme des substances?

3. L'usage de la terminologie pluraliste/moniste se trouve chez T. Schmaltz, « Descartes on Extension of Space and Time », Analytica, Rio de Janeiro, vol. 13, n. 2, (2009), p. 118.

4. AT, VII, 44.

5. « Par exemple, si une pierre est mue, et avec cela carrée, nous pouvons connaître sa figure carrée sans savoir qu'elle soit mue, et réciproquement nous pouvons savoir qu'elle est mue sans savoir si elle est carrée ; mais nous ne pouvons avoir une connaissance distincte de ce mouvement et de cette figure si nous ne connaissons qu'ils sont tous deux en une même chose, à savoir en la substance de cette pierre ». AT, VIII, 29-30.

6. «Ainsi la main est une substance incomplète, si vous la rapportez à tout le corps dont elle est partie; mais si vous la considérez toute seule, elle est une substance complète». AT, IX, 173. «Et comme celui qui dirait que le bras d'un homme est une substance réellement distincte du reste de son corps, ne nierait pas pour cela qu'il est de l'essence de l'homme entier, et que celui qui dit que ce même bras est de l'essence de l'homme entier, ne donne pas pour cela occasion de croire qu'il ne peut pas subsister par soi ». AT, IX, 177.

7. «Mais il n'en est pas ainsi de la substance que nous considérons sous la forme d'un os, et de celle que nous considérons sous la forme de chair : ce qui fait que nous ne pouvons pas les prendre pour une même chose en unité de nature, mais seulement en unité de composition, en tant que c'est un même animal qui a de la chair et des os ». AT, IX, 227. 
Et, finalement, dans l'Examen du Susdit Placard, il désigne les habits d'un homme de la même façon ${ }^{8}$. Prenant en compte ces textes, quelques interprètes, tels que Vere Chappell, Dan Kaufman, Paul Hoffman et Edward Slowik, soutiennent que pour Descartes les corps sont des substances.

Cependant, cette lecture peut devenir problématique si l'on considère l'extrait de l'Abrégé des Méditations où Descartes semble suggérer, contrairement aux textes précédents, qu'il existe une seule substance corporelle dans la nature dont les parties seraient des déterminations modales ${ }^{9}$. Dans cet extrait, Descartes commence par une description des substances qui sont créées par Dieu. Ces substances sont décrites comme ayant une nature incorruptible, raison pour laquelle elles peuvent être appelées de pures substances. Descartes affirme alors que le corps «pris en général » est une substance de ce type. Ensuite, il oppose ce corps au corps humain, et il distingue le corps humain des autres corps particuliers. L'opposition entre le corps «pris en général » et le corps humain repose sur l'incorruptibilité des parties dans le premier cas, et dans la corruptibilité des parties dans le second. Encore dans cette perspective, Descartes oppose la corruptibilité du corps humain à l'incorruptibilité de l'âme. Finalement, il affirme que le corps "pris en général » et les âmes seraient des substances pures et, dans ce sens, incorruptibles; tandis que le corps humain serait corruptible et sujet à des changements de leurs parties. Le fait que Descartes exclue le corps humain de la catégorie de substance pure, et affirme que seul le corps «pris en général » est une substance de ce type, suggère que pour lui les corps particuliers ne sont pas des substances. Par conséquent, il n'existe qu'une seule substance corporelle dans la nature dont les parties sont des déterminations modales.

8. «Enfin il faut remarquer ici que dans les sujets qui sont composés de plusieurs substances, souvent il y en a une qui est la principale, et qui est tellement considérée que tout ce que nous lui ajoutons de la part des autres n'est à son égard autre chose qu'un mode, ou une façon de la considérer ; ainsi un homme habillé peut être considéré comme un certain tout composé de cet homme, et de ses habits ; mais être habillé, au regard de cet homme, est seulement un mode, ou une façon d'être sous laquelle nous le considérons, quoique ses habits soient des substances ». AT, VIII, 351.

9. «[...] premièrement, afin de savoir que généralement toutes les substances, c'est-à-dire (toutes) les choses qui ne peuvent exister sans être créées de Dieu, sont de leur nature incorruptibles, et ne peuvent jamais cesser d'être, si elles ne sont réduites au néant par ce même Dieu qui leur veuille dénier son concours ordinaire. Et ensuite, afin que l'on remarque que le corps, pris en général, est une substance, c'est pourquoi aussi il ne périt point ; mais que le corps humain, en tant qu'il diffère des autres corps, n'est formé et composé que d'une certaine configuration de membres, et d'autres semblables accidents ; et l'âme humaine, au contraire, n'est point ainsi composée d'aucuns accidents, mais est une pure substance. Car encore que tous ses accidents se change, par exemple, qu'elle conçoive de certaines choses, qu'elle en veuille d'autres, qu'elle en sente d'autres, etc., c'est pourtant toujours la même âme ; au lieu que le corps humain n'est plus le même, de cela seul que la figure de quelquesunes de ses parties se trouve changée. D'où il s'ensuit que le corps humain peut facilement périr, mais que l'esprit, ou l'âme de l'homme (ce que je ne distingue point), est immortelle de sa nature ». AT, VII, 13. 
Une réponse possible à cette lecture, que l'on peut trouver chez Vere Chappel1 $^{10}$, Dan Kaufman ${ }^{11}$, Paul Hoffman ${ }^{12}$ et Edward Slowik ${ }^{13}$, c'est la compréhension selon laquelle, dans l'Abrégé des Méditations, les corps ne seraient pas des substances pures et incorruptibles, comme l'esprit et la totalité de l'univers étendu, mais des substances impures et corruptibles. En revanche, Tad Schmaltz ${ }^{14}$, Daniel Garber ${ }^{15}$ et Matthew Stuart ${ }^{16}$ ont vraiment pris au sérieux le critère d'incorruptibilité requis par Descartes dans l'Abrégé pour l'attribution de substantialité. En considérant ce critère assez fort pour exclure le fait que les entités qui ne le possèdent pas soient conçues comme des substances, ils ont exclu par conséquent les corps particuliers de la catégorie cartésienne de substance créée, bien qu'ils aient sauvegardé la thèse pluraliste, comme nous le verrons ensuite.

Le premier point que Schmaltz souligne dans son analyse, c'est qu'il n'est pas totalement clair que le corps «pris en général» de l'Abrégé des Méditations se réfère à la totalité de l'univers étendu. Il argumente que dans la lettre à Mesland du 9 février 1645 Descartes utilise une expression semblable pour se référer à une partie délimitée de l'étendue, à savoir, l'expression le corps «en général ${ }^{17}$. Cependant, il ajoute que l'expression le corps «pris en général» de l'Abrégé n'a pas le même signifié que l'expression semblable de la lettre à Mesland. Selon Schmaltz, tandis que dans la lettre à Mesland Descartes soutient que le corps «en général » est corruptible; dans l'Abrégé il établit clairement une distinction entre l'incorruptibilité du corps «pris en général» et la corruptibilité du corps humain, ce qui empêche que ces expressions, bien que semblables dans les deux textes, signifient la même chose ${ }^{18}$.

10. V. Chappell, «Descartes'on substance», in Blackwell Companion to Descartes, J. Broughton, J. Carriero (dir.), Oxford, Blackwell, 2007, p. 260. V. Chappell, «L'homme cartésien », in Descartes : Objecter et répondre, J.-M. Beyssade, J.-L. Marion (dir.), Paris, Presses Universitaires de France, 1994, p. 408-410.

11. D. Kaufman, «Descartes on Composites, Incomplete Substances and Kinds of Unity », Archiv Für Geschichte der Philosophie, Berlin, vol. 90, n. 1, (2008), p. 65.

12. P. Hoffman, «The Unity of Descartes' Man», in René Descartes. Critical Assessments, G. J. D. Moyal (dir.), London, Routledge, 1991, 3 vol., p. 173.

13. E. Slowik, «Descartes and Individual Corporeal Substance», British Journal for the History of Philosophy, London, vol. 9, n. 1, (2001), p. 7.

14. T. Schmaltz, op. cit., p. 113-147.

15. D. Garber, «La Physique Métaphysique de Descartes», traduit de l'américain par S. Bornhausen, Paris, Presses Universitaires de France, 1999.

16. M. Stuart, «Descartes's extended Substances », in New Essays on the Rationalists, R. Gennaro, C. J. Huenemann (dir.), Oxford, Oxford University Press, 1999. p. 82-104.

17. « [...] quand nous parlons d'un corps en général, nous entendons une partie déterminée de la matière, et ensemble de la quantité dont l'univers est composé [...]». AT, IV, 166.

18. «That the "body taken in general" mentioned in the Synopsis is distinct from the corruptible parts of matter mentioned in the Mesland letter is clear from the stress in the former text on the fact that body in general, in contrast to the human body, is incorruptible ». T. Schmaltz, op. cit., p. 121. 
Selon Schmaltz, l'expression le corps «pris en général » de l'Abrégé des Méditations se réfère à une étendue spécifique actuelle, qui est incorruptible $^{19}$. Dans ce sens, cette étendue s'oppose au corps humain qui, bien qu'il soit une étendue spécifique actuelle, est corruptible. La suggestion de Schmaltz est que cette étendue spécifique actuelle et incorruptible de l'Abrégé soit comprise comme se référant à une pluralité de parties substantielles réellement distinctes. Ces parties seraient différentes des corps particuliers, dans le sens où les corps seraient individualisés par le mouvement, susceptibles de changements et donc corruptibles, comme c'est le cas du corps humain, tandis que les parties ne seraient pas individualisées par le mouvement, ni soumises à des changements, car elles seraient incorruptibles.

L'interprétation de Schmaltz suggère, par conséquent, que les parties de l'étendue sont distinctes des corps particuliers. Bien que Descartes affirme dans plusieurs textes que les pierres, les habits, la main et le bras d'un homme sont des substances, Schmaltz propose que ces entités soient des sujets substantiels seulement dans le sens où elles sont composées de sujets encore plus simples, qui sont eux-mêmes des substances, dans le sens qui entraîne l'incorruptibilité. Ainsi, dans la tentative de concilier le texte de l'Abrégé des Méditations, qui représente une forte base textuelle pour la défense de la thèse moniste, avec une lecture pluraliste de la substance corporelle cartésienne, Schmaltz admet l'existence de parties substantielles dans l'étendue, des parties qui enveloppent l'incorruptibilité requise pour le concept de substance dans ce texte. Garber partage aussi avec Schmaltz l'interprétation selon laquelle les substances corporelles pour Descartes ne sont pas la même chose que les corps particuliers ${ }^{20}$. De la même manière, Stuart propose que les substances corporelles soient identifiées avec la quantité de matière qui compose les corps particuliers ${ }^{21}$. Ce concept de

19. «The "body in general" of the Synopsis thus can be positioned between the "extension in general" of the Principles and "a body in general" of the Mesland letter. Body in the Synopsis shares with the extension of the Principles the fact that it does not depend for its identity on a certain kind of actual motion, and thus is distinguishable from the body in general in the Mesland letter. Yet the body of the Synopsis is also similar to the body of the Mesland letter in possessing a specific extension that can exist apart from thought. Both are therefore distinct from the generic extension of the Principles, which is a mere mental entity that is individuated by thought. In the Synopsis, then, Descartes attempts to make room for parts of matter that have an extra-mental existence but that differ from the particular bodies that are individuated, and can be corrupted, by motion ». T. Schmaltz, op. cit., p. 123.

20. «Des substances matérielles, si tant est qu'elles existent, ne sont pas la même chose que des corps individuels ». D. Garber, op. cit., p. 273.

21. «Descartes identifies extended substances with quantities of matter, things that can survive any rearrangement or scattering of their parts, but not the annihilation of any of them... Ordinary physical objects come into and go out of existence with the rearrangement of quantities of matter [...] but quantities of matter themselves can be created or destroyed only by God. Since every part of a quantity of matter is itself a quantity of matter, we also get the desired result that every part of an extended substance is itself an extended substance ». M. Stuart, op. cit., p. 99. 
«quantité de matière », proposé par Stuart, a été reconnu par Schmaltz comme désignant la même chose que le concept de «parties substantielles de l'étendue ${ }^{22}$. Jusqu'à présent, comme nous l'avons vu, les interprétations proposées par Schmaltz, Garber et Stuart prétendent rendre compatible l'extrait de l'Abrégé avec une lecture pluraliste. Ces interprètes pensent qu'il est possible de maintenir la thèse selon laquelle Descartes aurait admis une pluralité de substances corporelles, même en admettant que les corps particuliers soient exclus de la catégorie cartésienne de substance créée.

Pour soutenir la thèse selon laquelle les parties de l'étendue ne sont pas des modes, mais sont elles-mêmes des substances réellement distinctes, les interprètes pluralistes trouvent des appuis textuels dans l'article 60 de la $1^{\text {ère }}$ partie des Principes, lorsque Descartes dit que les parties de l'étendue peuvent être délimitées pour nous, dans notre pensée, comme des substances ${ }^{23}$; dans l'article 55 de la $2^{\mathrm{e}}$ partie des Principes, lorsqu'il considère les parties d'un corps solide comme des substances ${ }^{24}$; dans $L e$ Monde, lorsqu'il reconnaît que chaque corps peut être divisé en parties extrêmement petites non visibles à nos yeux ${ }^{25}$; dans la lettre à Gibieuf du 19 janvier 1642, lorsqu'il affirme explicitement que deux moitiés d'une partie de l'étendue, aussi petites soient-elles, sont des substances ${ }^{26}$; mais surtout dans un extrait des Réponses aux Sixièmes Objections, où il dit que les parties de l'étendue sont différentes des modes. Cela permet aux pluralistes de soutenir que les parties sont des déterminations substantielles, tandis que les modes sont de simples variations dans ces parties.

22. «Stuart's conclusion that Descartes identifies extended substances with quantities of matter is similar to my own ». T. Schmaltz, op. cit., p. 122, n. 22.

23. «C'est pourquoi, de ce que nous avons maintenant l'idée par exemple, d'une substance étendue ou corporelle, bien que nous ne sachions pas encore certainement si une telle chose est à présent dans le monde, néanmoins, parce que nous en avons l'idée, nous pouvons conclure qu'elle peut être, et qu'en cas qu'elle existe, quelque partie que nous puissions déterminer de la pensée doit être distincte réellement de ses autres parties ». AT, VIII, 28.

24. «Et je ne crois pas qu'on puisse imaginer aucun ciment plus propre à joindre ensemble les parties des corps durs, que leur propre repos. Car de quelle nature pourrait-il être ? Il ne sera pas une chose qui subsiste de soi-même ; car toutes ces petites parties étant des substances, pour quelle raison seraient-elles plutôt unies par d'autres substances, que par elles-mêmes ? Il ne sera pas aussi une qualité différente du repos, parce qu'il n'y a aucune qualité plus contraire au mouvement qui pourrait séparer ces parties, que le repos qui est en elles. Mais, outre les substances et leurs qualités, nous ne connaissons point qu'il y ait d'autres genres de choses ». AT, IX, 94-95.

25. «Pensez que chaque corps peut être divisé en parties extrêmement petites. Je ne veux pas, ajoute-t-il, déterminer si leur nombre est infini ou non ; mais du moins il est certain qu'à l'égard de notre connaissance, il est indéfini, et que nous pouvons supposer qu'il y en a plusieurs millions dans le moindre petit grain de sable qui puisse être aperçu de nos yeux ». AT, XI,12.

26. «Car de cela seul que je considère que les deux moitiés d'une partie de matière, tant petite qu'elle puisse être, comme deux substances complètes, et quarum idea non redduntur a me inadequatae per abstractionem intellectus, je conclus certainement qu'elles sont réellement divisibles ». AT, III, 473. 


\section{Quelques difficultés de l'interprétation pluraliste}

On peut trouver des variations dans l'interprétation pluraliste de la substance corporelle cartésienne. Le fait que l'on puisse considérer avec quelques pluralistes que des corps particuliers sont des substances peut impliquer soit que le texte de l'Abrégé des Méditations, où Descartes nie que de telles entités soient des substances, ne représente pas sa vraie position et doit, par conséquent, être lu comme un texte de faible valeur métaphysique, soit que Descartes aurait admis l'existence de degrés de substantialité, c'està-dire que les corps particuliers occuperaient un degré plus faible par rapport à la totalité de l'univers étendu. Ils seraient des substances impures en opposition aux substances pures, catégorie dans laquelle la totalité de l'univers étendu serait incluse.

La première alternative peut être considérée comme « incompatibiliste », dans le sens où elle ne rend pas le texte de l'Abrégé des Méditations compatible avec la thèse selon laquelle les corps particuliers sont des substances; la seconde alternative peut être considérée comme «compatibiliste», et est adoptée par Dan Kaufman, Paul Hoffman et Edward Slowik. D'autre part, admettre avec Tad Schmaltz, Daniel Garber et Matthew Stuart que les parties de l'étendue qui composent les corps particuliers sont les seules entités qui satisfont la catégorie de substances corporelles cartésiennes, implique de proposer une troisième alternative interprétative et, en outre, permet d'harmoniser le texte de l'Abrégé avec les autres textes où Descartes semble défendre plus clairement une position pluraliste.

En tenant compte de ces différentes positions pluralistes, nous mettrons en évidence quelques difficultés sous la forme d'arguments qui visent à les réfuter. Ces difficultés sont liées à quatre thèses de la physique cartésienne : (1) la négation de l'atome, (2) la négation du vide, (3) l'explication relationnelle de la superficie d'une partie de l'étendue et (4) la mutuelle dépendance des parties de l'étendue. Chacune de ces thèses, comme nous le verrons en détail, aura un rôle important à jouer dans la présentation des arguments contre l'interprétation pluraliste.

\section{(1) La négation de l'atome}

La thèse de la négation de l'atome apparait dans de nombreux textes de Descartes $^{27}$. Parmi eux, le plus important est l'article 20 de la $2^{\mathrm{e}}$ partie des Principes où Descartes explique pourquoi il ne peut pas y avoir d'atomes ou de petites parties indivisibles dans la matière ${ }^{28}$. Ce qui nous intéresse dans cette thèse, c'est le fait qu'elle représente une objection à la vision pluraliste

27. Cf. Principes, I, article 26, lettre à Mersenne du 15 avril 1630 et lettre à Gibieuf du 19 janvier 1642. AT, VIII, 14-15. AT, I, 139-140. AT, III, 476-477.

28. AT, VIII, 51-52. 
de la substance corporelle cartésienne. Sur la base de cette discussion, on pourrait argumenter que la reconnaissance par Descartes de la divisibilité indéfinie des parties de l'étendue, qui constitue un élément important en faveur de la thèse de la négation des atomes, pourrait impliquer la corruptibilité de ces parties. Selon cette lecture, la divisibilité des parties de l'étendue serait assimilée à sa corruptibilité. Le problème est que l'un des critères fournis par Descartes pour reconnaître qu'une entité est une pure substance est justement son incorruptibilité, comme cela ressort clairement de l'Abrégé des Méditations. Si les parties de l'étendue sont divisibles indéfiniment, alors elles sont corruptibles. Ainsi, cela semble suggérer que les parties de l'étendue ne sont pas des substances pour Descartes.

Cependant, on pourrait remettre en question l'association de la divisibilité indéfinie avec la corruptibilité en attirant l'attention sur le fait que Descartes considère aussi la substance corporelle indéfinie comme divisible, bien qu'il n'admette pas qu'elle soit corruptible. Le problème est que l'identification de la divisibilité indéfinie avec la corruptibilité pour exclure la substantialité des corps particuliers implique qu'ils ne soient pas des substances, mais implique aussi que la substance corporelle indéfinie ne soit pas une substance créée. C'est-à-dire que, bien qu'il soit clair que Descartes n'admet pas l'existence d'atomes dans la nature, car l'étendue est indéfiniment divisible, il n'est pas clair que la divisibilité indéfinie de l'étendue implique sa corruptibilité et, par conséquent, que les corps particuliers ou les parties de l'étendue ne sont pas des substances créées.

Dans l'Abrégé des Méditations, Descartes affirme que le corps humain, dans la mesure où il diffère d'autres corps, est formé et composé d'une configuration de membres et d'autres accidents. Dès lors, quand ce corps subit des modifications de ses parties, il n'est plus le même corps, c'est-àdire qu'il périt et se corrompt. Mais toujours dans ce texte, bien que Descartes considère que la substance corporelle indéfinie soit divisible, il ne soutient pas que sa divisibilité entraîne sa corruptibilité, comme dans le cas précédent. Au contraire, il affirme que cette substance est incorruptible. Ainsi, dans l'Abrégé, Descartes admet que la divisibilité du corps humain, par modification de ses parties, entraîne sa corruptibilité, mais il n'admet pas qu'il en aille de même quant à la divisibilité de la substance corporelle indéfinie.

En prenant en considération cette précision, l'argument en question peut être reformulé dans les termes suivants: d'une part, les corps particuliers sont indéfiniment divisibles, donc il n'y a pas d'atomes dans la nature. De plus, la divisibilité indéfinie de ces corps entraîne la corruptibilité, mais aucune substance cartésienne ne peut être corruptible, donc les corps particuliers ne sont pas des substances. D'autre part, le corps « pris en général » est indéfiniment divisible, donc il n'y a pas d'atomes dans la nature. Mais la divisibilité indéfinie de ce corps n'entraîne pas sa corruptibilité, donc il est une substance corporelle divisible, mais non corruptible. Ainsi, la divisibilité indéfinie des corps particuliers et du corps «pris en général » entraîne qu'il n'y a pas d'atomes dans la nature, 
mais seule la divisibilité indéfinie des corps particuliers entraîne la corruptibilité.

Cependant, comme nous l'avons vu, Schmaltz, Garber et Stuart soutiennent que seules des parties de l'étendue sont des substances corporelles. Pour ces interprètes, les corps particuliers sont corruptibles, mais pas les parties de l'étendue. Selon eux, la thèse qui apparaît dans l'Abrégé des Méditations, selon laquelle la divisibilité indéfinie dans le particulier entraîne la corruptibilité et, par conséquent, l'exclusion de substantialité, bien qu'elle s'applique aux corps particuliers, ne s'applique pas aux parties de l'étendue. Selon cette lecture, la substance corporelle indéfinie est divisible, mais elle n'est pas corruptible ; les corps particuliers sont divisibles mais ils sont corruptibles; des parties de l'étendue sont divisibles, mais elles ne sont pas corruptibles. Dans cette perspective, il est possible de conclure que l'argument selon lequel la divisibilité indéfinie de l'étendue entraîne sa corruptibilité est valide pour réfuter la position d'un interprète pluraliste qui défend que les corps particuliers sont des substances, mais pas celle d'un interprète pluraliste qui défend que seules des parties de l'étendue sont des substances corporelles. Dans ce dernier cas, le critère d'incorruptibilité, requis pour toutes les substances cartésiennes, est satisfait.

\section{(2) La négation du vide}

Dans plusieurs textes, Descartes nie l'existence du vide dans la nature ${ }^{29}$. Dans l'article 16 de la $2^{\mathrm{e}}$ partie des Principes, en argumentant qu'il ne peut pas exister d'attributs du néant et qu'il ne peut pas exister d'espace où il n'y a point de substance, il conclut à l'impossibilité du vide ${ }^{30}$. Cependant, une conséquence de l'interprétation pluraliste est qu'elle doit présupposer que si les parties de l'étendue sont des substances créées, alors elles doivent être réellement distinctes les unes des autres. Le problème est que consentir qu'une partie soit claire et distinctement conçue sans l'autre partie, et ainsi qu'une partie ne dépend pas de l'autre pour sa constitution, semble impliquer l'existence d'un espace vide entre ces deux parties ${ }^{31}$. Ce que Descartes refuse clairement.

29. Cf. AT, VIII, 44-45. AT, VIII, 45. AT, VIII, 45. AT, VIII, 46. AT, VIII, 46-47.

30. AT, VIII, 49.

31. Ce problème a été mis en évidence par Spinoza, qui a affirmé, dans la scolie de la proposition 15 de la $1^{\text {ère }}$ partie de l'Éthique, que ceux qui nient le vide doivent admettre que l'extension ne peut pas être composée de parties réellement distinctes : «Puisque donc il n'y a pas de vide dans la nature (là-dessus, voir ailleurs), mais que toutes les parties concourent nécessairement en sorte qu'il n'y ait pas de vide, de là suit aussi que ces mêmes parties ne peuvent pas réellement se distinguer, c'est-à-dire, que la substance corporelle, en tant qu'elle est substance, ne peut se diviser ». Éthique I, scolie de la proposition 15. B. Spinoza, Éthique, présenté et traduit par Bernard Pautrat, Éditions du Seuil, Paris, 1999. 
Pour échapper à cette difficulté, on peut remettre en question le fait que les parties de l'étendue soient des substances réellement distinctes, ceci impliquant l'existence d'un espace vide entre elles. Si tout ce qui possède une longueur, une largeur et une profondeur est une substance corporelle pour Descartes, alors ce qui est appelé vide serait aussi une substance corporelle. Si tel est le cas, le fait que les parties de l'étendue soient réellement distinctes n'entraine pas de vide entre elles, car Descartes considère que le «vide » serait une substance corporelle. Par conséquent, l'argument basé sur la négation cartésienne du vide devient seulement un problème apparent parce qu'il ne représente plus une objection réelle pour un interprète qui souhaiterait défendre une position pluraliste sur la substance corporelle.

\section{(3) L'explication relationnelle de la superficie d'une partie de l'étendue}

Dans l'article 15 de la $2^{\mathrm{e}}$ partie des Principes, Descartes explique que la superficie est un mode partagé par un corps et par les corps qui l'environnent : « [...] par la superficie, on ne doit entendre aucune partie du corps qui environne, mais seulement l'extrémité qui est entre le corps qui environne et celui qui est environné, qui n'est rien qu'un mode ou une façon $[\ldots] »{ }^{32}$. Le point principal de cette explication est que si la superficie est un mode partagé par un corps et par d'autres corps environnants, alors un corps doit être entouré d'autres corps pour qu'il puisse avoir une superficie limitée et être une partie finie de l'étendue. Descartes défend ainsi l'interdépendance des superficies des parties de l'étendue. Le problème est que l'interdépendance ne semble pas être un critère satisfait par une substance créée cartésienne. D'un côté, si les superficies des parties de l'étendue sont interdépendantes, ces parties ne sont pas des substances, car les substances créées sont indépendantes de toutes les autres substances, sauf de Dieu. D'un autre côté, si les superficies des parties de l'étendue ne sont pas interdépendantes, alors il y a du vide entre elles, ce que Descartes n'admet pas.

En réponse à ces difficultés, on peut argumenter que la superficie étant le mode d'une partie de l'étendue, le fait qu'elle soit un mode, et pour cela dépendante d'autres parties, n'est pas un problème. Il est clair que l'interdépendance des superficies des parties de l'étendue n'est pas un problème, car les superficies sont des modes et non des substances. Pourtant, pour qu'une substance soit une substance corporelle particulière, c'est-à-dire une partie déterminée de l'étendue, elle doit posséder une superficie qui la fasse dépendre du voisinage d'autres parties. Car s'il n'est pas possible de concevoir une partie de l'étendue sans sa superficie, alors le recours à l'argument de l'interdépendance des parties de l'étendue reste valide pour problématiser la thèse selon laquelle les parties de l'étendue sont des

32. AT, VIII, 47. 
substances créées cartésiennes, même en reconnaissant que la superficie est seulement un mode. De cette manière, une fois le problème de l'existence du vide entre deux substances corporelles réellement distinctes éliminé, se pose le problème de l'explication relationnelle des superficies des parties de l'étendue.

\section{(4) La mutuelle dépendance des parties de l'étendue}

S'il est possible d'admettre que, pour Descartes, l'étendue possède des parties substantielles, de telles parties doivent être conçues comme dépendantes de toutes les autres parties qui composent la totalité de l'étendue. Le problème de la mutuelle dépendance des parties de l'étendue consiste dans le fait que les parties incorruptibles de la matière, mentionnées dans l'Abrégé des Méditations, semblent dépendre de toutes les autres parties incorruptibles de l'étendue et, de cette façon, il semble que la substantialité de ces parties soit ébranlée. Pour résoudre ce problème, une suggestion proposée par Schmaltz est que le concept cartésien de distinction réelle, lorsqu'il est appliqué aux substances corporelles, soit compris de façon distincte de quand il est appliqué à différentes substances pensantes, tout comme quand il est pris pour distinguer des substances pensantes de substances corporelles $^{33}$. Dans le cas des substances corporelles, Schmaltz suggère que la distinction réelle ne doit pas présupposer que ces substances puissent exister séparées les unes des autres, comme dans les deux autres cas, mais seulement qu'on puisse les concevoir comme différents sujets de certains modes de l'étendue qui sont réellement distincts d'autres sujets d'autres modes de l'étendue ${ }^{34}$.

33. «Descartes is clear on the necessity of mutual separability in the case where the distinct substances have different kinds of attributes [...]. But Descartes also seems to be committed to the necessity of separability in the case of the real distinction of two created substances that share the attribute of thought. For surely he would conclude from the fact that two finite minds are really distinct that each can exist without the other [...]. However, this argument for the necessity of separability for the real distinction cannot apply to bodies [...]. No portion of specific extension can be single and complete apart from all other created substances, since all such portions are composed of distinct substantial parts from which they cannot exist in separation. In order to hold that each portion of specific extension is a substance that is really distinct from the parts that compose it, then, Descartes must employ a concept of the real distinction that, in contrast to the concept that applies to the case of created minds, does not require the possibility of existence apart from all other substances of the same type. But this difference in the understanding of the real distinction carries with it a difference in the concepts of substance that apply to minds and bodies ». T. Schmaltz, op. cit., p. 126-127.

34. « The question now is whether this whole could be something really distinct from the parts that compose it. The answer to this question depends on a crucial ambiguity in Descartes's remarks concerning the distinctio realis, that is, the real distinction between different substances. The account of this distinction in the Principles begins with the claim that substances are really distinct just in case "we can clearly and distinctly understand one apart from the other" (PP I.60, AT 8-1:28). On one reading, the claim is that we can so understand one to be a subject of properties that differs from other subjects. This reading 
Cependant, si le statut du mode chez Descartes est sa dépendance à une substance créée, et si le statut des substances corporelles, selon Schmaltz, est leur dépendance vis-à-vis d'autres substances corporelles, puisqu'elles ne peuvent pas être conçues comme pouvant exister séparées d'autres substances corporelles, alors dans quelle mesure seraient-elles différentes des modes ? Est-ce que cet affaiblissement de la substantialité des parties de l'étendue ne serait pas suffisant pour vider complètement le sens du concept cartésien de substance créée, qui se distingue du concept cartésien de mode ? Est-ce que le sens du concept de distinction réelle que Schmaltz propose entre différentes substances corporelles ne pourrait pas être conçu comme une distinction modale ? En tenant compte de ces difficultés, il nous reste à vérifier si elles rendent l'interprétation pluraliste de la substance corporelle cartésienne moins avantageuse que l'interprétation moniste.

\section{L'interprétation moniste comme issue}

Selon l'interprétation moniste, il y a une seule substance corporelle dans l'univers physique cartésien, dont la nature se réduit à une seule étendue géométrique, uniforme et homogène en toutes ses parties. Par conséquent, les corps particuliers qui composent le monde qui nous entoure sont des modes de cette seule substance. Les bases textuelles de cette interprétation, comme nous les avons indiqués précédemment, sont l'extrait de l'Abrégé des Méditations et aussi un fragment d'une lettre à Villebressieu de l'été 1631. Avec quelques nuances, l'interprétation moniste est partagée par plusieurs interprètes de Descartes, à savoir, Octave Hamelin ${ }^{35}$, Henri Gouhier ${ }^{36}$, Martial Gueroult ${ }^{37}$, Jean-Marie Beyssade ${ }^{38}$, Michelle Beyssade ${ }^{39}$, John

seems to be confirmed by the appeal in this passage to the fact that he can think of each part of corporeal substance as the subject of certain modes of extension that differs from subjects of other modes of extension. On this reading, it seems that we can think of the whole as really distinct from the parts that compose it, since the whole and its parts can be conceived as distinguishable subjects ». Ibid., p. 125.

35. «L'étendue sans limites est en même temps douée d'une très forte unité. Étant substance, elle est le plein, et, si elle n'est pas indivisible, les parties qu'elle admet sont toutes solidaires. Et Descartes reconnaît dans l'Abrégé des Méditations [...] qu'il n'y a qu'une seule substance matérielle dont les corps soi-disant individuels ne sont que des modes ». O. Hamelin, Le système de Descartes, Paris, Alcan, 1921, p. 305.

36. «Ce qui est substance et de ce fait impérissable, c'est la matière en général [in genere] et mon âme [mens] prise dans son individualité. Quant à ce morceau particulier de matière qu'est mon corps, il n'est qu'un certain arrangement de parties ». H. Gouhier, La pensée métaphysique de Descartes, Paris, Librairie Philosophique J. Vrin, 1999, p. 392.

37. «[...] il n'existe en réalité qu'une seule substance étendue dont elles [des substances physiques particulières] ne sont que des modes ». M. Gueroult, Descartes selon l'ordre des Raisons, Paris, Aubier, 1953, vol. I, p. 105.

38. «Le principe de la réponse est sans doute qu'un corps humain, comme corps, ne constitue en aucun sens une substance mais un assemblage (que ce soit de modes ou de parties). S'il n'y 
Cottingham $^{40}$, Roger Stuart Woolhouse ${ }^{41}$ et Alice Sowaal ${ }^{42}$. Bien que ces interprètes croient que pour Descartes il existe une seule substance corporelle dans la nature, et que les corps sont les modes de cette unique substance, ils admettent aussi qu'un degré plus faible de substantialité puisse être attribué à ces corps.

Gueroult soutient que pour Descartes les corps sont des substances par rapport aux autres corps, parce qu'on peut concevoir chaque corps indépendamment d'autres corps. Par exemple, on peut penser la cire comme réellement distincte de la pierre, bien qu'elles soient des modes par rapport à la substance corporelle, c'est-à-dire au corps «pris en général», parce qu'elles ne peuvent pas exister indépendamment de cette substance ${ }^{43}$. Selon Gueroult, ce qui permet qu'un corps en particulier soit considéré comme une substance est le fait qu'il conserve un invariant numérique déterminé, qui peut être conçu comme la subsistance d'une même quantité de matière à travers la diversité de ses aspects géométriques ${ }^{44}$. Cependant, Gueroult

a pas de troisième substance, c'est d'abord parce qu'en l'occurrence il n'y en a pas de seconde ». J.-M. Beyssade, Études sur Descartes, Paris, Éditions du Seuil, 2001, p. 242, n. 53. 39. «Les corps particuliers, y compris le corps d'un homme, sont des parties de l'unique substance corporelle ou étendue, des parties du corps pris en général ». M. Beyssade, «Le dualisme cartésien et l'unité de l'homme », in L'Esprit Cartésien: Actes du XXVI ${ }^{\mathrm{e}}$ Congrès de l'Association des Sociétés de Philosophie de Langue Française, B. Bourgeois, J. Havet (dir.), Paris, Librairie Philosophique J. Vrin, 2000. p. 2.

40. « Descartes oferece uma perspectiva radicalmente monística da substância corpórea [...]. As coisas individuais, - os planetas, os cavalos, as árvores - são assim compostos simplesmente porque são modificações pontuais de uma substância simples e extensa ». J. Cottingham, A filosofia de Descartes, trad. M. R. S. Guedes, Rio de Janeiro, Edições 70, 1989, p. 119.

41. «The idea then seems to be that unlike human minds, human bodies (as also stones or horses) are not numerically different individual substances, but pieces of corporeal substance or body as such, in the way that pieces of lead are just that - pieces of lead and not lead's ». R. S. Woolhouse, Descartes, Spinoza, Leibniz. The concept of substance in seventeenth century metaphysics, New York, Routledge, 1993, p. 22.

42. «I argue that this approach is flawed because individual bodies are not secondary substances ». A. Sowaal, «Cartesian Bodies », Canadian Journal of Philosophy, Canada, vol. 34, n. 2, Texas, (2004), p. 223.

43. «En ce qui concerne les corps, nous concevons de telles substances lorsque nous pouvons penser clairement et distinctement chacune d'elles, en excluant de leur idée tout le reste de la substance étendue. Par exemple, ce qui fonde pour moi la substance singulière de tel corps, comme la cire ou la pierre, c'est que je puis penser clairement et distinctement telle partie de l'étendue en excluant d'elle toutes les autres parties [...]. Dans ces conditions, un certain mode de la substance universelle des corps, lui-même diversifié par une infinité de modes subalternes peut être considéré à son tour comme substance en opposition avec les autres modes de cette substance que nous rejetons hors de ce mode pour en avoir une connaissance claire et distincte. Ainsi la cire, la pierre, sont, d'un côté, de simples modes de l'étendue, mais d'un autre côté, chacun de ces modes (par exemple, la cire, mode étendu considéré comme unité de divers modes ou propriétés qui sont ceux de la cire) apparaît comme une substance par rapport à tout le reste de l'étendue ». M. Gueroult, op. cit., p. 105-106.

44. «Immutabilité d'un mode de l'étendue qui, lui-même, peut présenter des modifications subalternes, cette substance de la cire n'est pas autre chose que la subsistance d'une certaine 
ajoute à son explication que cet invariant numérique, qui permet qu'un corps particulier déterminé soit considéré comme une substance, est seulement apparent, parce que pour Descartes les corps n'ont en fait pas d'indépendance par rapport à la substance corporelle, et ne sont donc pas des substances créées au sens fort et strict, mais seulement des modes d'une seule substance corporelle ${ }^{45}$.

Gueroult défend alors la thèse selon laquelle il y aurait des degrés de substantialité dans la métaphysique cartésienne. Ainsi, selon lui, Descartes aurait admis une seule substance de premier ordre qui serait Dieu, deux types de substances de second ordre qui seraient les substances pensantes et la substance corporelle, et plusieurs substances de troisième ordre qui seraient les corps particuliers ${ }^{46}$. Cependant, le concept de substance de troisième

quantité d'étendue sous la diversité de ses aspects géométriques. Bref, l'unité et l'identité d'un corps physique, grâce à quoi nous l'identifions comme étant telle substance, c'est la capacité qu'il possède de conserver une même quantité sous divers aspects, gagnant en largeur ce qu'il perd en profondeur ou en épaisseur, et inversement [...]. La substance particulière d'un corps n'est donc rien d'autre qu'un invariant numérique qui le fait, - dans cette mesure - indépendant du reste, et qui le constitue comme principe d'explication autonome des divers aspects qu'il revêt». Ibid., p. 106-107. "La thèse de l'invariant numérique conçu comme subsistance du même volume total à travers la diversité de déformations ne s'applique qu'aux substances agrégats, qui d'ailleurs seules sont visibles, par exemple, à ce morceaux de cire ou à ce morceaux de fer. En effet, chaque corpuscule ou molécule d'un corps donné garde à la fois son volume et sa forme. L'invariance numérique de la substance agrégat n'est que la subsistance en lui des mêmes molécules en même quantité, quels que soient les figures et les états de cette substance, tant que cette substance n'est pas détruite ». M. Gueroult, op. cit., p. 107, n. 163.

45. «On trouve dans cette application indistincte du concept de la substance tant aux substances universelles créées, étendue et pensée, qu'aux substances particulières étendues, qui sont en réalité des modes ». Ibid., p. 108-109. «Les substances corporelles, ayant des parties, sont sans fondement intrinsèque, et, si elles ne se détruisent pas en fait, restent par nature exposée à la destruction. Si elles se maintiennent, ce n'est pas par soi, c'est en vertu des lois physiques et du jeu de l'ensemble des modes. Ainsi, d'une part, la subsistance de l'invariance numérique permet de penser, tant qu'elle persiste, tel corps, en excluant de lui tous les autres modes distincts de lui et de le concevoir par là comme indépendant, c'est-àdire comme substance, d'autre part, la physique, révélant que cette persistance elle-même dépend du concours mécanique de l'ensemble des modes de la substance étendue, assure que l'indépendance de telle substance corporelle à l'égard des autres modes n'est au fond qu'apparente. Toutefois, la stabilité de fait présentée par les invariants numériques nous autorise à concevoir les corps d'espèces différentes à la façon de substances et de les traiter comme s'ils étaient absolument tels. Mais ce sont là substances du troisième ordre, substances selon l'opinion commune, non substances du deuxième ordre, ou substance à la rigueur ». Ibid., p. 115.

46. «En réalité, Dieu est le seul digne de la substantialité, parce qu'il est le seul être à se concevoir par soi au sens absolu du terme, étant le seul être à se causer et à se soutenir par soi. Il peut être sans l'étendue, tandis que celle-ci ne peut être sans lui. Toutefois, une substance créée, quoique ne pouvant être conçue absolument par soi, puisqu'elle dépend de Dieu qui la cause et la soutient, n'a besoin d'aucune autre substance créée pour être et être conçue. Elle peut être conçue sans ses modes, non ceux-ci sans elle. Elle est donc, dans cette mesure, conçue par soi, et peut être investie à ce titre d'une substantialité de second ordre. À leur tour, les substances particulières ne sauraient être des substances dans le sens où l'est l'étendue, car 
ordre se réfère, dans la vision de Gueroult, à une compréhension peu stricte du sens du terme «substance » pour Descartes, de telle façon que ce n'est que sous certaines conditions que l'on pourrait appeler un corps de substance de troisième ordre ${ }^{47}$.

Bien que les substances de second ordre - les âmes et le corps «pris en général »-soient dépendantes dans le sens causal de la substance de premier ordre - Dieu -, elles ne dépendent pas les unes des autres ni d'autres choses créées dans ce sens. Mais quoique les substances de troisième ordre ne dépendent pas dans le sens causal d'autres substances de troisième ordre, elles dépendent dans le sens d'inhérence de la substance corporelle de second ordre. Ainsi, le fait que les substances corporelles de troisième ordre - les corps - soient dépendantes dans le sens d'inhérence de la substance corporelle de second ordre - le corps «pris en général »-permet aussi de considérer les corps comme des modes de cette substance. De cette façon, les conditions sous lesquelles Gueroult admet l'existence de substances corporelles de troisième ordre dans la métaphysique de Descartes sont compatibles avec une interprétation moniste.

Quoique Descartes n'ait pas explicité ni développé la terminologie des substances des premier, second et troisième ordres, nous pensons qu'elle peut être utile pour expliquer pourquoi il appelle substance des choses qu'il ne comprend pas réellement comme des substances créées dans sa métaphysique. C'est le cas, par exemple, de son affirmation dans la Troisième Méditation que la pierre est une substance. Si Descartes ne considère pas réellement que la pierre soit une substance, alors pourquoi la désigne-t-il comme substance ? La réponse que nous proposons à cette difficulté textuelle repose sur la notion de substance de troisième ordre. Cette notion se réfère à une compréhension peu stricte du sens du terme substance créée chez Descartes. Par substances de troisième ordre, nous comprenons des choses qui sont inhérentes à la substance corporelle de second ordre. C'est le cas par exemple de la pierre, de la main, du bras et des vêtements d'un homme. De cette façon, nous pensons que la notion de substance de troisième ordre, qui se réfère aux modes de la substance corporelle de second ordre, a la fonction de sauvegarder une lecture moniste et d'expliquer ainsi la raison de certaines usages imprécis du terme substance dans les textes de Descartes.

elles en sont des modes et elles ne peuvent être conçues sans celle-ci, tandis que celle-ci peut être conçue sans elles. Mais elles peuvent être investies d'une substantialité de troisième ordre, en tant que, tout en étant des modes, elles n'ont pas besoin des autres modes de la substance universelle à laquelle elles se rapportent pour être conçues clairement et distinctement ». M. Gueroult, op. cit., p. 109.

47. En fait, pour Gueroult, une substance corporelle de troisième ordre (un corps) n'est qu'un mode de la substance corporelle de second ordre (du corps «pris en général»). «On comprend dans ces conditions que Descartes puisse réserver le nom de substance stricto sensu aux âmes individuelles, naturellement indestructibles de par leur indivisibilité, et à la substance étendue en général qui, seule, et contrairement aux substances corporelles particulières, est naturellement impérissable ». Ibid., p. 115. 


\section{Une difficulté inhérente à l'interprétation moniste}

La lecture moniste semble faire face à une difficulté lorsque nous avons à l'esprit la thèse métaphysique selon laquelle seules des substances créées peuvent être sujets ultimes d'inhérence de propriétés dans la philosophie de Descartes, dont la conséquence est le fait que toute propriété doit toujours se référer en fin de compte à une substance créée. Le problème qui surgit ici est le suivant: si le corps humain est un mode d'une substance corporelle, alors il ne peut pas être le sujet ultime d'inhérence de propriétés. Si c'est le cas, des propriétés purement corporelles, que Descartes identifie comme le premier degré de réponse sensorielle, dans les Réponses aux Sixièmes Objections $^{48}$, ne pourraient pas avoir comme sujet ultime d'inhérence le corps humain, puisqu'il ne serait pas une substance de ce type, mais un mode de cette substance. Par conséquent, de telles propriétés seraient apparemment des propriétés d'une seule entité substantielle qui serait la totalité de l'univers étendu. Le problème est que cela peut sembler être, à première vue, un contre-sens.

Ainsi, lorsque l'on voit un bâton, les mouvements qu'il produit dans nos nerfs optiques, et qui produisent des mouvements dans notre cerveau, seraient des propriétés de la totalité de l'univers étendu et non d'un corps humain en particulier. De la même manière, des mouvements purement corporels, qui produisent des changements dans les organes des animaux, seraient des propriétés de la totalité de l'univers étendu, et non des animaux en particulier ${ }^{49}$. Ces deux cas attirent notre attention sur la difficulté à soutenir la thèse selon laquelle toute propriété doit présupposer une substance créée comme sujet ultime d'inhérence, à partir d'une lecture moniste de la substance corporelle. En aucun cas, comme cela apparait clairement, le corps humain ou l'animal ne seraient des sujets ultimes d'inhérence de propriétés, puisqu'ils ne tombent pas sous le concept de substance créée.

Une réponse possible à cette objection serait d'argumenter en faveur du fait que les corps peuvent être des sujets d'inhérence de propriétés purement corporels, même s'ils ne sont pas des sujets ultimes, car seules les substances

48. « Pour bien comprendre quelle est la certitude du sens, il faut distinguer en lui trois sortes de degrés. Dans le premier, on ne doit considérer autre chose que ce que les objets extérieurs causent immédiatement dans l'organe corporel; ce qui ne peut être autre chose que le mouvement des particules de cet organe, et le changement de figure et de situation qui provient de ce mouvement ». AT, XI, 236-237.

49. Comme nous l'avons vu, dans la distinction des trois degrés de réponse sensorielle des Réponses aux Sixièmes Objections, Descartes caractérise le premier degré par des mouvements purement corporels qui peuvent être trouvés aussi bien dans un corps humain que dans un animal. Voir aussi sur ce point la lettre à Morus du 5 février 1649 : «Et cela, d'abord parce qu'il est certain que dans le corps des animaux, ainsi que dans les nôtres, il y a des os, des nerfs, des muscles, du sang, des esprits animaux, et autres organes disposés de telle sorte qu'ils peuvent produire par eux-mêmes, sans le secours d'aucune pensée, tous les mouvements que nous observons dans les animaux ». AT, V, 277. 
créées peuvent occuper cette place. Dans la lettre à Mersenne (pour Hobbes) du 21 avril 1641, Descartes affirme qu'un accident peut être le sujet d'un autre accident, selon ses mots : "Car il n'y a point d'inconvénient ou d'absurdité à dire qu'un accident soit le sujet d'un autre accident, comme on dit que la quantité est le sujet des autres accidents $»^{50}$. Ainsi, rien n'empêche qu'une propriété purement corporelle ait pour sujet d'inhérence le corps d'un humain ou celui d'un animal, à condition que le corps en question, qui est un mode, ait pour sujet ultime d'inhérence la substance corporelle dont il est un mode, à savoir, le corps « pris en général ».

Une autre réponse possible à cette objection consiste à arguer du fait que si les corps peuvent être conçus comme des substances corporelles de troisième ordre, alors les variations perçues dans ces corps, c'est-à-dire les propriétés purement corporelles de ces corps, en seraient les modes, même de troisième ordre. Cependant, qui veut soutenir une position moniste n'a pas nécessairement besoin de soutenir qu'il y ait un troisième degré de substantialité dans les corps, il suffit de les considérer simplement comme des modes de la substance corporelle de second ordre. En effet, comme Descartes l'explique à Hobbes, les modes peuvent être les sujets d'autres modes. Ainsi, la thèse selon laquelle les corps auraient, pour ainsi dire, une substantialité de troisième ordre pourrait être acceptée par ceux qui veulent soutenir cette position et respecter à la lettre tous les textes de Descartes, car, strictement parlant, la lecture moniste ne reconnaît pas une vraie substantialité aux corps.

En tous les cas, nous sommes d'accord avec l'interprétation moniste dans le sens où le sujet ultime d'inhérence de propriétés purement corporelles est une seule substance corporelle, qui possède une diversité de modes, à savoir, les corps. D'une part, ces modes peuvent être des sujets d'inhérence d'autres modes, mais pas des sujets ultimes. D'autre part, ces modes peuvent aussi être considérés comme des substances corporelles de troisième ordre, et ainsi posséder des modes et qualités de troisième ordre. De cette façon, et à partir de ce qui a été dit jusqu'ici, nous pensons que la lecture moniste n'est pas incompatible avec les principes de la métaphysique et de la physique de Descartes, contrairement à ce que nous avons constaté dans le cas de la lecture pluraliste, et est totalement compatible avec son dualisme.

Avant de finir, il convient de faire une remarque sur le cas particulier du corps humain. Comme nous l'avons vu, ce corps peut être sujet d'inhérence de propriétés corporelles, à condition qu'il soit dépendant d'un sujet encore plus élémentaire et ultime d'inhérence, qui est la substance corporelle de second ordre. De la même façon, animaux, horloges, fontaines artificielles, moulins et orgues d'églises, dans la mesure où ils sont des modes de la substance corporelle de second ordre, peuvent aussi être sujets d'inhérence de propriétés corporelles. Cependant, bien que tous ces corps possèdent le 
même statut ontologique de modes d'une seule substance corporelle de second ordre, il faut remarquer que Descartes reconnait dans quelques textes la spécificité du corps humain par rapport aux autres corps ${ }^{51}$. Dans ces textes, il affirme que l'une des marques de cette spécificité est le fait que le corps humain a été programmé par Dieu pour être uni avec l'âme. Néanmoins, certains problèmes découlent de cette reconnaissance du caractère particulier du corps humain, problèmes liés à l'absence de l'indivisibilité réelle chez les animaux et à la question de savoir si Descartes aurait admis une vision hylémorphique dans son anthropologie, par exemple. L'examen de ces problèmes dépasse la portée de cet article. De toute façon, nous pouvons souligner que la spécificité du corps humain par rapport aux autres corps est liée à une forme spéciale d'organisation et de fonctionnement, ainsi qu'à la relation intime d'union qu'il entretient avec l'âme.

\section{Bibliographie :}

Beyssade, J.-M., Études sur Descartes, Paris, Éditions du Seuil, 2001.

Beyssade, M., «Le dualisme cartésien et l'unité de l'homme »; in L'Esprit Cartésien: Actes du XXVI ${ }^{\mathrm{e}}$ Congrès de l'Association des Sociétés de Philosophie de Langue Française, B. Bourgeois, J. Havet (dir.), Paris, Librairie Philosophique J. Vrin, 2000.

Chappell, V., «Descartes' on substance», in Blackwell Companion to Descartes, J. Broughton, J. Carriero (dir.), Oxford, Blackwell, 2007, p. 251-270.

Chappell, V., "L'homme cartésien », in Descartes : Objecter et répondre, J.-M. Beyssade, J.-L. Marion (dir.), Paris, Presses Universitaires de France, 1994, p. 403-426.

Cottingham, J., A filosofia de Descartes, trad. M. R. S. Guedes, Rio de Janeiro, Edições 70, 1989.

Descartes, R., CEuvres Philosophiques de Descartes en 3 volumes, F. Alquié (éd.), Paris, Garnier, 1997.

Descartes, R., Euvres de Descartes en 12 volumes, C. Adam et P. Tannery (éd.), Paris, Librairie Philosophique J. Vrin, 1996.

Garber, D., La Physique Métaphysique de Descartes, trad. de l'américain par S. Bornhausen, Paris, Presses Universitaires de France, 1999.

Gueroult, M., Descartes selon l'ordre des Raisons, Paris, Aubier, 1953, vol. I.

51. Lettre à Regius de décembre 1641. AT, III, 460-461. Lettre à Chanut du $1^{\text {er }}$ février 1647. AT, IV, 604-605. Lettre à Mesland du 9 février 1645. AT, IV, 166-167. 
Gouhier, H., La pensée métaphysique de Descartes, Paris, Librairie Philosophique J. Vrin, 1999.

Hamelin, O., Le système de Descartes, Paris, Alcan, 1921.

Hoffman, P., «The Unity of Descartes' Man », in René Descartes. Critical Assessments, G. J. D. Moyal (dir.), London, Routledge, 1991, 3 vol. p. 168-193.

Kaufman, D., « Descartes on Composites, Incomplete Substances and Kinds of Unity », Archiv Für Geschichte der Philosophie, Berlin, vol. 90, n. 1, (2008), p. 40-72.

Schmaltz, T., «Descartes on Extension of Space and Time », Analytica, Rio de Janeiro, vol. 13, n. 2, (2009), p. 113-147.

Skirry, J., Descartes and the Metaphysics of Human Nature, London, New York, Continuum, 2005.

Slowik, E., «Descartes and Individual Corporeal Substance», British Journal for the History of Philosophy, London, vol. 9, n. 1, (2001), p. 1-15.

Sowaal, A., « Cartesian Bodies », Canadian Journal of Philosophy, Canada, vol. 34, n. 2, Texas, (2004), p. 217-240.

Spinoza, B., Éthique. Présenté et traduit par Bernard Pautrat. Paris, Éditions du Seuil, 2010.

Stuart, M., «Descartes's extended Substances », in New Essays on the Rationalists, R. Gennaro, C. J. Huenemann (dir.), Oxford, Oxford University Press, 1999. p. 82-104.

Woolhouse, R. S., Descartes, Spinoza, Leibniz. The concept of substance in seventeenth century metaphysics, New York, Routledge, 1993. 